(Article)

\title{
Evaluation of Column Test Methods to Study Inactivation of Chemically Bonded Silica Gels
}

Toshihiko Hanai*

Health Research foundation, Reserch Institute for Production Development 4F, Sakyo-ku, Kyoto, 606-0805 Japan *

hanai104@kf7.so-net.ne.jp; 81-45-295-1468

\author{
Received: date; Accepted: date; Published: date
}

\begin{abstract}
The silanol activity and trace metal detection methods for chemically bonded silica gels were evaluated in silico. Test compounds with large molecular sizes may demonstrate negative results because of the possibility of indirect hydrogen bonding via short alkyl groups or siloxane of the silica gels. This hypothesis was based on the observation of weak hydrogen bonding energy values similar to those observed in the study of the alkyl group effect on the hydrogen bonding of alkanols. Consequently, smaller molecules may be a better choice for the analysis of bonded-phase quality.
\end{abstract}

Keywords: inactivation column test; silanol, trace metals, in silico, bonded-phase silica

\section{Introduction}

Since their inception by Kirkland octadecyl-silanized silica gels have been the main packing materials in liquid chromatography [1]. The introduction of pure silica gel improved the quality of bonded-phase silica gels [2], and various quality test methods have been proposed and discussed [3]. The chromatographic behavior of high-pKa bases helped identify the different characteristics of bonded-phase silica gels. Pyridine was found to be a more sensitive compound than aniline for silanol detection, and the peak shape was affected by the components of the eluent [4]. A column evaluation methodology was described for the chromatography of basic compounds, and it was found that the chromatograms obtained using marketed columns varied for the compounds tested with different eluents $[5,6]$. Several columns were tested for reversed-phase liquid chromatography were tested, and the physicochemical interactions were qualitatively described, and the results suggested the necessity of individual characterization [7]. A method for the selection of a column for the chromatographic separation of basic compounds was proposed [8]; however, a description of fundamental properties of bonded phase silica gels was lacking. A significant amount of metallic impurities is generally present in the ordinary silica gels and they severely affected the activity of free silanol groups. It was found that washing the gels with an acid significantly reduced the negative effects of metal impurities [3,9]. Therefore, a specific column test is required for general use. A new type of packing material was introduced for reversed-phase liquid chromatography and the performance was evaluated by comparing it with an ordinary octadecyl bonded silica gel [10]. Further test methods need to be developed to study its long-term stability with a good peak shape for a variety of compounds.

Generally, the inactivation process is necessary to obtain stable and reproducible packing materials, especially for basic compounds, wherein the nitrogen containing groups can easily form hydrogen bonding with the silanol groups of the gels. Typically, the short alkyl groups such as trimethyl-silane are bonded to the free silanol groups as secondary siliration, whereas the larger ligands are incapable of completely covering the silanols because of the restrictions in physical 
contact. The atomic distance between the silanol groups is usually greater (5.37 $\AA$ ) than that between the chloro or alkoxyl groups of silylation reagents (3.43 and $3.01 \AA$, respectively) [11]. Currently, manufacturers use pure silica gels, the purity is over 5 nine. Iron, calcium, aluminum, sodium, and magnesium are the coomon trace metals found in silica gels, with the source of the metals being the water used in the sieving process. The careful management of the reaction processes produces chemically inert bonded-phases and ensures a symmetrical peak shape and as well as over two months of operation for quality control analyses [12]. However, the quality of the marketed packed columns is not uniform, and the manufacturers demonstrate the quality of the gels using specific chromatograms. The column test compounds are classified into two groups. One set ofcompounds is employed to test the presence of unreacted silanol groups, while the other set is used to evaluate the purity of silica gels, which can have trace metal inputities. However, experimental demonstration is not straightforward and consequently, quantitative assessment is performed by in silico analysis using the model phases. Previously, the effect ofalkyl-chain lengths on bonded silica gels have been studied. It was observed that up-to a length of the butyl-chain, the silica gel was affected by silicon oxide and therefore, the analysis supported the instability of the butyl- bonded phase silica gel. The in silico analysis successfully demonstrated the electron localization and atomic partial charge of the end carbon in the butyl group [13]. Thus, a similar approach was applied using model alkyl-phases from methyl to hexyl-phases. The solutes used here are aniline, pyridine, amitriptyline,

8-hydroxyquinoline, quinolizaline, and hinokitiol.

Initially, aniline was used to detect the presence of silanol groups based on the acid-base interaction. Subsequently, pyridine was used because of its smaller molecular size and higher sensitivity for the detection of silanols. Silica gels with a purity of $>99.999 \%$ were introduced and they are, currently, the common support materials for analytical columns as they have a guaranteed chemical stability and produce symmetrical peak shapes. Such evolution in the packing materials helps support long-term reproducible quality control in liquid chromatography. Crytical test methods have been proposed to further improve the long term operation of these materials. Amitriptyline is a compound that demonstrated a poor peak shape from those columns that had previously demonstrated an excellent peak in the case of pyridine. The molecular mass of amitriptyline is higher than that of pyridine. The quation then arises whether amitriptyline can access the free silanol groups that could not be accessed using pyridine. Ideally, amitriptyline should not be capable of interactions with the free silanol groups, but this may indicate a poor surface coverage of the alkyl-ligands.

8-hydroxyquinoline, quinolizaline, and hinokitiol are chelate reagents, and they are used to detect trace metals. Chemically bonded silica gels from pure silica gels should not contain any surface trace metals. However, above mentioned compounds exhibited a tailing peak shape. This may be due to the presence of trace metals from solvents including water that cancontaminate the silica gel and remain on the surface of the bonded phases. On the other hand, the tailing peak shape may aldo be a result of an indirect interaction of the compounds with the silica gels as was demonstrated in the case of the alkyl-chain effect of butyl bonded silica gels. The latter case was studied by in silico analysis using model bonded phases.

\section{Experimental}

A model dimethyl-alkyl mono-chlorosilane bonded phase was constructed as shown in Figure 1. The connection of silicon dioxide using silicon atoms was eliminated, and silicon trioxide was locked as a part of silica gel. The model phase size was determined to minimize the calculation time and the molecular size of the analytes. The alkyl groups produce a tightly densed phase. In reality such bonded phases are not synthesized using porous silica gels; however, such phases are used to analyze the indirect interaction between an analyte and a model phase. The bonded alkyldimethylsilane was kept free to enable contact of the analyte with the alkyl groups. The methyl group was changed to ethyl and so on upto hexyl to study the effects of the alkyl chain length. 


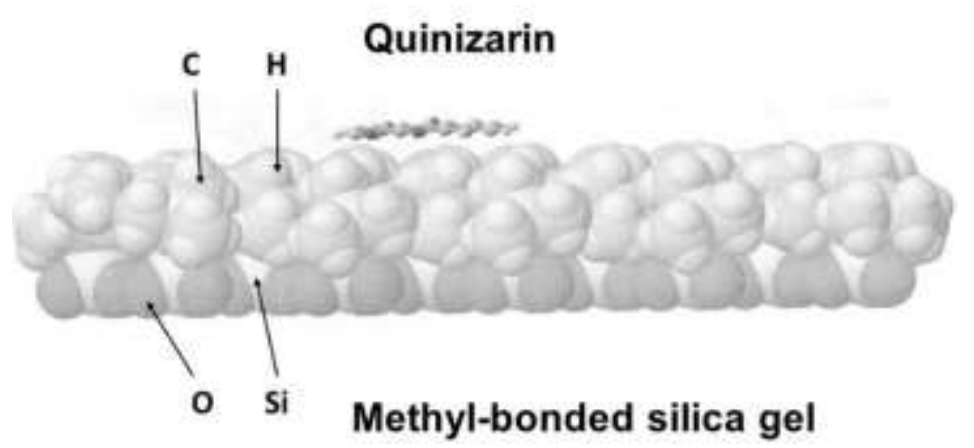

Figure 1. Adsorption of quinizarin on methyl-bonded silica gel phases

These molecular interaction (MI) energy values $\left(\mathrm{kcal} \mathrm{mol}^{-1}\right)$ are the sum of a solute and model phase energy values minus a complex energy value, calculated as per the following equations [11]. MIHB, MIES, and MIVW are MI energy of hydrogen bonding (HB), electrostatic (ES), and van der Waals (VW) energy values.

$\mathrm{MIHB}=\mathrm{HB}$ (molecule A) + HB (molecule B) - HB (molecule A and molecule B complex),

MIES = ES (molecule A) + ES (molecule B) - ES (molecule A and molecule B complex),

and

MIVW $=$ VW (molecule A) + VW (molecule B) - VW (molecule A and molecule B complex). The relative MIHB, MIES, and MIVW values indicate the contribution level.

\section{Results and discussion}

The alkyl groups are condensed in the maximum bonded phase to avoid the presence of any free silanol groups. Therefore, all the analytes can interact only with the alkyl groups and not the silanols. The analytes may have indirect contact with silicon dioxide through the surface of the alkyl phase. If the alkyl phase is completely hydrophobic, the molecular interaction between an analyte and the model phase should indicate MIVW and not MIHB and MIES [11]. The properties of the standard compounds are listed in Table 1.

Table 1. Properties of standard compounds

\begin{tabular}{ccc}
\hline Chemicals & $\log P$ & $\mathrm{pKa}$ \\
\hline $\begin{array}{c}\text { For silanol activity } \\
\text { Pyridine }\end{array}$ & 0.70 & 5.23 \\
Amitriptyline & 4.92 & 9.40 \\
For trace metals & & \\
Quinolinol & 1.72 & - \\
Hinokitiol & 2.10 & - \\
Quinizarin & 3.70 & - \\
\hline
\end{tabular}

The docking process was followed. Analyte at a distance greater than $6 \AA$ from the model phase were located and then, the structure was optimized. The MI energy was calculated using the above equations. The calculated MI energy values are summarized in Table 2. 
Table 2. Molecular interaction energy values of analytes with model alkyl-bonded silica gels

\begin{tabular}{|c|c|c|c|c|c|c|c|}
\hline Chemicals & MIHB & MIES & MIVW & Chemicals & MIHB & MIES & MIVW \\
\hline Model phases & & & & Model phases & & & \\
\hline Methyl phase & & & & Butyl phase & & & \\
\hline Pyridine & 0.000 & 0.068 & 6.635 & Pyridine & 0.000 & -0.027 & 0.724 \\
\hline Amitriptyline & 0.000 & 0.091 & 25.652 & Amitriptyline & 0.000 & -0.024 & 21.443 \\
\hline Quinolinol & 0.015 & 0.121 & 22.40 & Quinolinol & 0.009 & 0.041 & 28.766 \\
\hline Hinokitiol & 0.092 & 0.199 & 11.718 & Hinokitiol & 0.000 & -0.056 & 34.453 \\
\hline Quinizarin & 0.147 & 0.272 & 8.613 & Quinizarin & 0.017 & -0.030 & 29.576 \\
\hline Ethyl phase & & & & Pentyl phase & & & \\
\hline Pyridine & 0.000 & -0.119 & 13.027 & Pyridine & 0.000 & 0.024 & 6.512 \\
\hline Amitriptyline & 0.000 & -0.209 & 33.890 & Amitriptyline & 0.000 & -0.146 & 9.169 \\
\hline Quinolinol & -0.001 & -0.114 & 26.551 & Quinolinol & 0.000 & -0.021 & 6.276 \\
\hline Hinokitiol & 0.011 & -0.105 & 11.505 & Hinokitiol & -0.001 & -0.179 & 12.431 \\
\hline Quinizarin & 0.041 & -0.176 & 27.315 & Quinizarin & 0.002 & -1.111 & 15.992 \\
\hline Propyl phase & & & & Hexyl phase & & & \\
\hline Pyridine & 0.000 & -0.028 & 8.759 & Pyridine & 0.000 & -0.098 & 18.709 \\
\hline Amitriptyline & 0.000 & -0.065 & 10.981 & Amitriptyline & 0.000 & 0.038 & 22.126 \\
\hline Quinolinol & 0.018 & 0.071 & 13.130 & Quinolinol & 0.003 & -0.038 & 23.970 \\
\hline Hinokitiol & 0.014 & -0.266 & 12.361 & Hinokitiol & 0.000 & 0.052 & 30.703 \\
\hline Quinizarin & 0.019 & -0.045 & 11.791 & Quinizarin & 0.000 & 0.009 & 26.339 \\
\hline
\end{tabular}

MIHB, MIES, MIVW: molecular interaction hydrogen bonding, electrostatic and van der Waals energy values between an analyte and a model phase, $\mathrm{kcal} \mathrm{mol}^{-1}$.

Pyridine and amitriptyline did not show any polar interaction with the methyl-phase. This implies that the methyl phase is mimics inactivated silica gel. However, quinolinol, hinokitiol, and quinizarin exhibited polar interactions, although metal atoms were not present on the surface. Such polar interactions ceased after interactions with the butyl-phase. This result suggested that although the surface modification is successfully performed based on the silanol activation test, the metal sensitive compounds may demonstrate the poor performance of the chromatogram peaks. The reason for this is not due to the presence of trace metals but due to the insufficient hydrophobicity of the modified surface. The alkyl-chain length effect on the hydrophobicity of alkyl-bonded silica gels was explained from the electron density map [13]. This result suggested that first, the silanization of silica is important. Second silanization cannot eliminate the poor chromatographic performance of metal sensitive compounds. If the chromatographic behavior of metal sensitive compounds is significant, the manufacturers should perform trace metal analysis of their products and then, analyze the quality of the reagents.

\section{Conclusion}

Some test compounds for the evaluation of the activity of untreated silanol moieties and the existence of trace metals may misguide the scientific explanation. Based on the in silico model analysis, it can be hypothesized that large and hydrophobic test compounds may interact with weak hydrogen bonding via the short alkyl groups or directly interact with siloxane. Therefore, a simple test using smaller molecules is recommended to evaluate the quality of bonded-phase silica gels.

Funding: Please add: “This research received no external funding”

Conflicts of Interest: Declare conflicts of interest or state "The authors declare no conflict of interest." 


\section{References}

1. Kirkland, J.J. DeStefano, J.H. Controlled Surface Porosity Supports with Chemically-Bonded Organic Stationary Phases for Gas and Liquid Chromatography, J. Chromatogr. Sci., 1770, 8 : 309-314.

2. Ohhira, M. Ohmura, F. Hanai, T. Inertness of bonded silica gel packings. J. Liq. Chromatogr., 1989, 12: 1065-1074.

3. T. Synthesis and properties of stable bonded siica gel packings and the performance, Hanai,T. Hatano, H. (eds) Advances in liquid chromatography, World Sci. Pub. Singapore, 1996, 307-329.

4. MaCally, D.V. Effect of organic modifier and nature of solute on the performance of bonded silica reversed-phase columns for the analysis of strong basic compounds by hogh-performance liquid chromatography, J. Chromatogr. A, 1996, 738: 169-179.

5. Mapihan, K.Le. Vial, J. Jardy, A. Reversed-phase liquid chromatography column testing: robustness study of the test, J. Chromatogr. A, 2004, 1061: 149-158.

6. Stella, C., Rudaz, S., Gauvrit, J.-Y., Lantéri, P., Huteau, A., Tchapla, A., Veuthey, J.-L. Characterization and comparison of the chromatographic performance of different types of reversed-phase stationary phases, $J$. Pharm. Biomed. Anal. 2007, 43: 89-98.

7. Mapihan, K.Le. Vial, J. Jardy, A. Reversed-phase liquid chromatography column testing and classification: Physicochemical interpretation based on a wide set of stationary phases, J. Chromatogr. A, 2007, 1144: 183-196.

8. MaCally, D.V., The challenges of the analysis of basic compounds by high performance liquid chromatography: some possible approaches for improved separations, J. Chromatogr. A, 2010, 1217: 858-880.

9. Gwarda, R.Ł., Szwerc, W., Aletańska-Kozak, M., Klimek-Turek, A., Torbicz, A., Chomicki, A., Kocjan, R., Matosiuk, D., Dzido, T.H. The influence of metallic impurities on the free silanol activity of commercial thin-layer chromatography adsorbents demonstrated by retention changes of basic/amphoteric compounds such as peptides, J. Planar Chromatogr. - Modern TLC, 2017, 30: 375-385.

10. Huo, Z., Chen, L. Base-deactivated and alkaline-resistant chromatographic stationary phase based on functionalized polymethylsilsesquioxane microspheres, J. Sep. Sci. 2020, 43: 389-397.

11. Hanai, T. Quantitative in silico chromatography: Computational modeling of molecular interactions, Royal Society of Chemistry, Cambridge, UK, 2014, (ISBN: 978-1-84973-991-7), 1-338

12. Hanai, T.; New developments in liquid chromatographic stationary phases, Advances in chromatography, 2000, 40: 315-357.

13. Hanai, T.; Fundamental properteis of packing materials for liquid chromatography, Separations 2019, http://creativecommons.org/lisenses.by/4.0/ 